\title{
Do somatic USP8, USP48 and BRAF mutations differ in their genotype-phenotype correlation in Asian Indian patients with Cushing's disease?
}

\section{Ananth P Abraham}

Christian Medical College Vellore

\section{Rekha Pai}

Christian Medical College Vellore

\section{Daniel L Beno}

Christian Medical College Vellore

\section{Geeta Chacko}

Christian Medical College Vellore

Hesarghatta Shyamasunder Asha

Christian Medical College Vellore

\section{Simon Rajaratnam}

Christian Medical College Vellore

Nitin Kapoor

Christian Medical College Vellore

Nihal Thomas

Christian Medical College Vellore

Ari George Chacko ( $\square$ arichacko@cmcvellore.ac.in )

Christian Medical College Vellore https://orcid.org/0000-0003-2757-2108

\section{Research Article}

Keywords: USP8, USP48, BRAF, Cushing's disease, corticotroph

Posted Date: June 18th, 2021

DOI: https://doi.org/10.21203/rs.3.rs-626247/v1

License: (c) (i) This work is licensed under a Creative Commons Attribution 4.0 International License. Read Full License 


\section{Abstract \\ Purpose}

To estimate the prevalence of USP8, USP48 and BRAF mutations in patients with Cushing's disease (CD) from the Indian subcontinent, and determine their genotype-phenotype correlation.

\section{Methods}

We prospectively recruited 46 patients with CD who underwent surgery between September 2015 and July 2019 at our institute. Fresh frozen tumour tissue was obtained in all patients. Using Sanger sequencing, the presence of somatic USP8 mutations was documented and the frequency of USP 48 and BRAF mutations in USP8 wild-type corticotroph adenomas was determined. Clinical, hormonal and surgical data were then compared between USP8, USP48- and BRAF-variant carriers and patients with wild-type tumours.

\section{Results}

Signature USP8 mutations were detected in 17 (37\%) patients. Of the 29 USP8 wild-type adenomas, 4 (13.8\%) harboured USP48 mutations, one of them being a splice-site mutation that has previously not been described. BRAF mutations were not found in any of the 29 patients. Corticotroph adenomas with USP8 mutations had a higher incidence of Crooke's hyaline change than wild-type tumours $(70.6 \% \mathrm{vs}$. $37.9 \%, p=0.032$ ). Adenomas with USP48 mutations had a higher rate of cavernous sinus invasion than their wild-type counterparts ( $50 \%$ vs. $4 \%, p=0.042$ ). No other significant phenotypic difference could be established between mutant and wild-type tumours.

\section{Conclusions}

The prevalence of USP8 mutations in our series of patients with CD was $37 \%$. The prevalence of USP48 mutations in USP8 wild-type adenomas was $13.8 \%$, including a novel splice-site mutation. BRAF mutations were not found in any USP8 wild-type tumour. USP8-mutants showed significantly more Crooke's hyaline change and USP48-mutants were more likely to demonstrate cavernous sinus invasion.

\section{Introduction}

Cushing's disease (CD) is a type of adrenocorticotrophic hormone (ACTH) dependant Cushing's syndrome (CS) caused by hypersecretion of ACTH by pituitary adenomas. Extensive research has been done to explore the possible molecular mechanisms responsible for it but the exact aetiopathogenesis remains unclear. 
Several recent publications have identified somatic mutations in the ubiquitin-specific protease 8(USP8) genes of $21-62 \%$ patients with CD.[1-5] These mutations in exon 14 of the USP8 gene, via increased deubiquitinase (DUB) activity, have been shown to increase the expression of epithelial growth factor receptors (EGFR), thereby up-regulating EGFR-induced proopiomelanocortin (POMC) transcription and ACTH secretion. While the link between EGFR signalling and USP8 mutations was established in in-vitro studies, $[1,3]$ other authors failed to demonstrate increased EGFR expression on immunohistochemistry (IHC) in USP8 mutated corticotroph adenomas.[6] A recent publication[7] addresses this by demonstrating that mutant adenomas also suffer dysregulation of a number of other proteins namely $\mathrm{p} 27 / \mathrm{kip} 1$, CABLES1, HSP90, and activated CREB; all of which have been previously found to be associated with either increased ACTH production or increased cell proliferation.[8]

Further research revealed that in USP8 wild-type corticotroph adenomas, mutations in USP48 (10-23\%) as well as $B R A F(1-16 \%)$, which are also known to increase POMC transcription, might be the drivers of ACTH over-production. $[9,10]$

What is evident from the literature is that there is a wide variation in the reported prevalence of USP8, USP48 and BRAF mutations from different countries. There is also limited clinical, hormonal and radiological data available on patients harbouring these mutations. Few studies have shown that USP8mutant corticotroph adenomas occur more frequently in women, are smaller in size, are less invasive, and are more likely to lead to disease remission following surgical resection.[2, 5, 6] A single study showed that USP48-variant adenomas were smaller in size.[10]

In this study we aimed to determine the prevalence of signature and novel mutations in these genes in a cohort of South Asian patients for the first time, and also look for definite genotype-phenotype correlation in terms of their clinical, hormonal and radiological profile.

\section{Materials And Methods}

\section{Design and setting}

This was a prospective study conducted at the Christian Medical College, Vellore with the collaborative efforts of the Departments of Neurosurgery, Endocrinology and Pathology. It was approved by the Research and Ethics Committee of the Institutional Review Board (IRB Min No: 9628, dated 01.09.2015). The design was cross-sectional, to estimate the frequency of somatic USP8 mutations in patients with CD and frequency of USP 48 and BRAF mutations in patients with USP 8 wild-type tumours.

\section{Clinical data}

Between September 2015 and July 2019, all patients diagnosed to have CD using standard clinical and biochemical criteria and who had discernible tumours on dedicated magnetic resonance imaging (MRI) sequences of the pituitary gland were enrolled in the study after obtaining informed consent in the case of adults, and assent in the case of minors. All patients underwent endoscopic transsphenoidal resection 
of their corticotroph adenomas. If patients were on ketoconazole, the drug was stopped 2 weeks prior to surgery. Using the surgical strategy previously published by us,[11] either selective adenomectomy, enlarged adenomectomy, hemihypophysectomy or subtotal tumour resection was done, depending on whether tumour margins were clearly defined intraoperatively. Perioperative steroids were not administered in order to enable early evaluation of remission. Postoperatively, patients were started on steroids only if their $8 \mathrm{AM}$ cortisol level dropped $<5 \mu \mathrm{g} / \mathrm{dl}$ or if they demonstrated symptoms of hypocortisolaemia or hyponatraemia. Patients were asked to review 3 months following surgery and thereafter every year. Remission was defined by either the presence of a basal cortisol of $<5 \mu \mathrm{g} / \mathrm{dl}$ with requirement for steroid replacement therapy, or a basal cortisol $>5 \mu \mathrm{g} / \mathrm{dl}$ but with suppression to $<1.8$ $\mu \mathrm{g} / \mathrm{dl}$ with the $1 \mathrm{mg}$ overnight dexamethasone suppression test

\section{Pathological evaluation and mutational analysis}

Each biopsy specimen obtained for a patient was equally divided for histopathological examination and genetic sequencing. Tissue for histopathological examination was fixed in $10 \%$ buffered formalin and embedded in paraffin. Standard haematoxylin-eosin and reticulin staining was employed. Disruption of the normal acinar architecture along with the presence of monomorphic cells was considered as confirmation of tumour. If tumour could not be identified on initial sections, serial sectioning and examination with both haematoxylin-eosin and reticulin staining was done. Periodic acid Schiff (PAS) staining and IHC for ACTH (1:150, Biogenex, San Ramon, CA), other anterior pituitary hormones, and MIB1 labelling index were performed on all surgical specimens using $5-\mu \mathrm{m}$ sections. PAS and cytokeratin immunostainings were also used to confirm Crooke's hyaline change in normal adenohypophysis.

DNA was extracted from the fresh frozen samples of tissue that was confirmed to be tumorous on histopathology. The samples, which were stored at $-70^{\circ} \mathrm{C}$, were first thawed and a standardised protocol for DNA extraction from tissues was followed. The QIAamp DNA Mini Kit (Qiagen) was used for all extractions. The DNA was quantitated using a NanoDrop ${ }^{\mathrm{TM}}$ microvolume spectrophotometer (NanoDrop Technologies) and the $260 / 280$ ratio was determined. Samples with a poor ratio or quantity were amplified with primers targeting a $133 \mathrm{bp}$ region of an endogenous control, before they were subjected to further analysis.

The polymerase chain reaction (PCR) for the two primer sets of exon 14 (USP8) were performed using previous published primers.[2] For USP48 and $B R A F$, the primer sequences used were based on that employed by Chen et al.[9] and Ferchichi et al. respectively.[12] Details of primer sequences and thermal cycling conditions are elaborated in Supplementary Table 1. All reactions were carried out in $25 \mu \mathrm{l}$ volume. The following thermal cycling profile was employed for all PCRs: $95^{\circ} \mathrm{C}$ for 8 minutes, $95^{\circ} \mathrm{C}$ for 30 seconds, optimized anneal for 30 seconds, $72^{\circ} \mathrm{C}$ for 1 minute and final extension of $72^{\circ} \mathrm{C}$ for 10 minutes. The PCR product was detected using a $1.5 \%$ agarose gel. Sanger sequencing of both the sense and antisense strands of products for both primer sets were performed with an automated DNA sequencer (ABI PRISM 310 genetic analyser) using the ABI PRISM BigDye Terminator Cycle Sequencing Ready 
Reaction Kit (Applied Biosystems). Mutational analysis was performed by comparing the sequence with the wild type and by looking for the presence of all known mutations in this exon.

\section{Data collection and statistical analysis}

A comprehensive proforma was used to record the clinical, biochemical and radiological parameters of each patient. Details of the surgery, histopathological examination, mutational analysis and postoperative complications were also documented. Follow-up data was regularly updated as and when patients reviewed in the outpatient clinic. Mean with standard deviation (SD) / median with inter-quartile range (IQR) were used for continuous variables as applicable. Frequency and percentage were used for categorical variables. Prevalence was expressed as a proportion with $95 \% \mathrm{Cl}$. Categorical variables were compared using Chi-square testing. Continuous variables were compared using Student's t-test if normal assumptions were satisfied; otherwise the Mann-Whitney $U$ test was used. Multivariate penalised logistic regression was done on variables which had $p$-values $<0.2$ on bivariate analysis. Statistical analysis was performed using IBM SPSS Statistics for Windows, version 21 (IBM Corp., Armonk, N.Y., USA) and Stata version 15 (StataCorp LLC, TX, USA).

\section{Results}

During the study period, a total of 49 patients were recruited for the study, however 3 patients were subsequently excluded because adequate fresh frozen tumour tissue was not available for DNA extraction.

\section{Preoperative data}

The mean age of our study population was $29.5 \pm 8.2$ (range 12-48) years and included 3 adolescents aged 12,13 and 15 years. Thirty eight (82.6\%) were females. Six (13\%) patients had been operated elsewhere earlier and presented to us with recurrent/persistent $C D$. The mean duration of symptoms was 2.6 (range $0.2-10$ ) years. Nineteen (41.3\%) patients were on preoperative ketoconazole for a mean duration of 2.5 (range $0.3-14$ ) months. Control of hypercortisolaemia with ketoconazole was achieved in only 2 out of these 19 patients.

There were 29 (63\%) microadenomas and 17 (37\%) macroadenomas. Of the macroadenomas, 13 were Wilson-Hardy Grade A tumours, 3 were Grade B and 1 was grade E. Four (8.7\%) adenomas showed cavernous sinus invasion. Table 1 summarises the preoperative data of all patients.

\section{Surgical details}

Gross total resection could be achieved in 44 (95.7\%) patients. Only subtotal resection was achieved in 2 $(4.3 \%)$ patients because of cavernous sinus invasion and bleeding that limited further resection. Of the patients who had gross total resection, 17 (37\%) underwent selective adenomectomy, 25 (54.3\%) underwent enlarged adenomectomy and 2 (4.3\%) underwent hemihypophysectomy. 
At 3 months follow-up, $37(80.4 \%)$ out of 46 patients were in remission. Only 4 out of $8(50 \%)$ males entered remission, while $33(86.8 \%)$ females achieved the same. Amongst those who went into remission, long term follow-up was available in 31 patients with a mean duration of $25.3 \pm 13.6$ (range 6-50) months. Seven $(22.6 \%)$ of these 31 patients ( 6 female and 1 male) subsequently had disease recurrence with a mean time to recurrence of $20.1 \pm 7.4$ (range 8-28) months.

\section{Mutational analysis}

In all 46 patients, the diagnosis of corticotroph adenoma was confirmed by histopathological examination and immunopositivity for ACTH. On DNA sequencing, 17 (37\%) patients had somatic mutations of the USP8 gene in the mutational hotspot of exon 14 at the 14-3-3 binding motif. Of these, 11 were missense mutations while the remaining were frameshift mutations. All mutations were located in the amino acids (AA) 718-720 with the most common being p.Pro720Arg and p.Ser719del fs. The full list of mutations is found in Supplementary Table 2. Figure 1(a) shows the DNA sequencing chromatogram of a USP8 wild-type tumour, Figure 1(b) shows the chromatogram of a USP8-mutant with a p.Pro720Arg mutation, and Figure 1(c) shows the chromatogram of frameshift mutation (p.Ser719del fs).

In the 29 USP8 wild-type adenomas, USP48 mutations were present in 4 (13.8\%), with three missense mutations (p.Met415lle) and1 splice-site mutation (p.Pro433IVS+2 T>A). There were no BRAF mutations in any of our patients. Figure 2(a) depicts the DNA sequencing chromatogram of patient with no USP48 mutation, Figure 2(b) is the chromatogram of a patient with a p.Met415lle mutation in exon 10 of the USP48 gene and Figure 2(c) shows a USP48 splice-site mutation (p.Pro433IVS+2 T>A).

\section{Genotype-phenotype correlation of patients with USP8 and USP48 mutations}

Tables 2 and 3 compare the clinical, radiological, biochemical and surgical characteristics of the USP8mutants and USP8 wild-type tumours. The only significant difference between both groups was an increased presence of Crooke's hyaline change in the adjoining hypophysis of the mutated tumours (70.6\% vs. $37.9 \%, p=0.032$ ). There was larger number of macroadenomas in the USP8-mutant group but this fell just short of statistical significance $(52.9 \%$ vs. $27.6 \%, p=0.085)$. Only 1 out of $8(12.5 \%)$ males harboured the mutation while 16 out of $38(42.1 \%)$ females had mutated tumours although this was not statistically significant. Also not statistically significant but important to note, was a higher rate of disease recurrence in patients with USP8-mutant tumours ( $33.3 \%$ vs. $18.2 \%, p=0.38)$. On multivariate analysis, Crooke's hyaline change remained significantly higher in patients with mutated tumours (OR $5.05,95 \% \mathrm{Cl} 1.02-25 ; p=0.047)$. All other variables were similar in both groups.

The comparison between patients with USP48-mutants and USP48 wild-type tumours is made using bivariate analysis in Tables 4 and 5 . The only significant difference between both groups was a higher rate of cavernous sinus invasion in the USP48-mutants ( $50 \%$ vs. $4 \%, p=0.042)$. Tumours with USP48 mutations recurred more frequently than their wild-type counterparts (33.3\% vs. $15.8 \% ; p=0.470)$, 
although this was also not statistically significant like in the case with the USP8-mutants. On multivariate analysis, there was no variable that was significantly different between the 2 groups.

\section{Discussion}

\section{Prevalence of USP8, USP48 and BRAF mutations}

The prevalence of USP8 mutations in CD reported thus far ranges from 21-62\% (Supplementary Table 3). $[1-6,13,14]$ Our study, the first from South Asia, found a prevalence of $37 \%$. The USP8 mutations found in our patients have been previously described and no novel mutations were discovered, however, we found a hitherto unreported USP48 splice-site mutation - p.Pro433IVS+2 T>A.

Somatic mutations of both USP48 and BRAF genes in patients with CD were reported only in 2018. $[9,10]$ The pooled incidence of USP48 and BRAF mutations in these two studies was $41 / 308(13.3 \%)$ and $16 / 227$ (7\%).[15] In our study, the prevalence of USP48 mutations in USP8 wild-type adenomas was $13.8 \%$, almost identical to that in the literature. Of interest is that almost all USP48-and BRAF-variants were noted only in patients with USP8 wild-type tumours, although they themselves were not mutually exclusive.

Another important finding in our study was that not a single BRAF mutation was detected in the 29 patients with USP8 wild-type status. The absence of BRAF mutations in our patients with $C D$ is in stark contrast to the $16.5 \%$ prevalence reported from China[9] but is similar to $1 \%$ prevalence amongst Caucasians.[10] Ethnicity may also have a bearing on the presence of USP8-variants, $[1,2,5,7,10,14]$ as is evident from the higher prevalence of USP8 mutations (62\%) in the Chinese population[3] as compared to that in the European population (21-48\%).

\section{Genotype-phenotype correlation of USP8-variants}

Several studies have shown a significantly higher, if not exclusive occurrence of USP8-variants in women. $[2,3,6,10,13,16]$ This was however not the case in the series by Albani et al.,[14] who found that there was no significant gender association (USP8 mutations in $39 \%$ of females vs. $30 \%$ of males). In our series, only 1 male out of $8(12.5 \%)$ had a USP8 mutation while 16/38 (42.1\%) females carried USP8-variants. No male patient out of the 4 with USP8 wild-type CD harboured USP48 mutations. While this difference did not attain statistical significance due to the relatively small sample size, there seems to be a clear tendency towards female preponderance and might explain, at least partially, the well-known female predilection in $\mathrm{CD}$ which so far has not been adequately understood. Perez-Rivas et al.[2] first proposed that oestrogens could demonstrate a growth-stimulating effect on USP8-mutated corticotrophs, a hypothesis supported by the fact while a balanced sex-ratio exists amongst paediatric patients with $\mathrm{CD}[17]$ in adults there is a distinct female predominance of the disease. ${ }^{11}$ In addition, the demonstration of oestrogen receptors on corticotroph cells along with the stimulatory effect of oestradiol on murine corticotroph cell proliferation via EGFR signalling might be a reason for a higher incidence of CD in females.[18,19] 
Some reports indicate that USP8-mutated tumours occur in younger adults $[2,7,14]$ and older children,[13] however, we and others[3-6] found no significant age difference between carriers of USP8-mutant and wild-type adenomas. BMI was found to be significantly higher in patients harbouring USP8-mutant tumours in two studies, $[2,10]$ while another found that BMI was lower amongst patients with USP8mutant tumours.[13] We could not demonstrate any statistically significant difference in BMI between the 2 groups.

We found that the USP8-mutants in our series tended to be larger than their wild-type counterparts, although this did not achieve statistical significance $(p=0.09)$. Findings on the issue of tumour size vary considerably, with variant-carrying tumours being reportedly smaller and less invasive in some studies, $[3,6]$ while others have observed a greater size in USP8-mutant microadenomas compared with wild-type microadenomas. [2,5] This difference may be at least partially attributed to a selection bias in some studies. For example, Hayashi and colleagues[6] oversampled Crooke's cell adenomas, while the Chinese series[3] included a relatively large proportion of invasive tumours $(>20 \%)$ and giant adenomas. Also worth noting is that none of the series reported so far mirror the ratio between micro- and macroadenomas normally found in $\mathrm{CD}$, i.e. less than $10-20 \%$ macroadenomas, probably a consequence of the need for adequate pathological specimens to perform DNA or RNA sequencing.

With regards to the biochemical characteristics of these patients, one study[2] found that patients with USP8 mutations demonstrate increased suppression of cortisol after $8 \mathrm{mg}$ dexamethasone while another found significantly lower plasma ACTH levels in these patients.[6] Ma et al.[3] found that ACTH secretion was higher in patients carrying USP8 mutations. We failed to establish any significant difference between wild-type and mutated tumours in the biochemical parameters studied, a finding corroborated by others. $[2,5,13,14]$

However, despite revealing no significant difference between the ACTH, UFC and serum cortisol in USP8variant and USP\& wild-type we did find that the proportion of patients with Crooke's hyaline change in the adjoining adenohypophysis was significantly higher in the USP8-variant group. Crooke's hyaline change is typically seen with higher levels of hypercortisolism,[20] so it may be conjectured that patients with USP8mutant adenomas may have experienced severe hypercortisolism at some point of their illness.

The remission rates of CD was similar in the USP8-variant and wild type tumors as reported by others. $[2,3,14]$ Some authors $[5,6]$ note a higher remission rate in patients with USP8 mutations while others $[13,14]$ report higher recurrence rates in mutated tumours. Another study found parallel recurrence rates, but a shorter mean time to recurrence in the USP8-variant adenomas.[3] We too found a higher recurrence rate amongst our patients with a USP 8 mutation (33\% vs. $18 \%$ ), although it was not statistically significant due to the small sample size.

\section{Genotype-phenotype correlation of USP48-variants}

The study by Chen et al.[9] could not demonstrate any significant clinical, biochemical or radiological differences between patients with USP48-variants and USP48 wild-type corticotroph adenomas. However 
a subsequent study reported that USP48-mutant adenomas were smaller than wild-type adenomas.[10] In our series, the occurrence of cavernous sinus invasion was significantly higher in the 4 patients with USP48-mutant adenomas ( $50 \%$ vs. $4 \%, p=0.042$ ). This difference has not been reported by any other study, however further research is required on these less common mutations before any meaningful conclusions can be drawn. The literature available on the genotype-phenotype correlation of CD patients with USP8 and USP48 mutations is summarised in Table 6.

\section{Strengths and limitations of this study}

This single-centre, prospective study from the Indian subcontinent, determines the prevalence of USP8, USP48 and BRAF mutations in patients with CD using fresh frozen tumour tissue that provided uniformity in the quality of tissue used for mutational analysis. The main limitation of the study was its relatively small sample size that precluded the establishment of statistically significant phenotypic differences between patients with USP8-/ USP48-variant tumours and their wild-type counterparts. Moreover, only mutations in the previously described mutational hotspots of the USP8, USP48 and BRAF genes were tested for. Next-generation sequencing (NGS) may have identified additional mutations in our series, as was seen in the study by Ballmann and colleagues,[4] where NGS detected a few USP8 mutations that were missed on Sanger sequencing.

\section{Conclusions}

The prevalence of somatic USP8 mutations in corticotroph adenomas was $37 \%$. The frequency of USP48 mutations in patients with USP8 wild-type adenomas was $13.8 \%$ while $B R A F$ mutations were not found in any of them. We report for the first time, a novel USP48 splice-site mutation (p.Pro433IVS + 2 T > A). USP8mutated tumours had an increased presence of Crooke's hyaline change in the normal adenohypophysis. Tumours with USP48 mutations had significantly more cavernous sinus invasion than USP48 wild-type adenomas.

\section{Declarations}

\section{Acknowledgements}

We would like to sincerely thank Dr. L Jeyaseelan and Ms. Malavika Babu from the Department of Biostatistics for their help with the statistical analysis of the data.

\section{Disclosure}

The findings of this study were presented at the annual conference of the Indian Society of NeuroOncology, 2021.

\section{Author contributions}


APA, AGC, RP, GC and HSA contributed to conception and design of the study. AGC operated on the patients in this study. GC supervised the histopathological examination of all tumours. DLB and RP did DNA sequencing of the tumor samples. HSA, SR, NT and NK were responsible for diagnosis of the patients with Cushing's disease and their medical management. APA collected patient information, performed statistical analysis and interpretation of data, and wrote the first draft of the manuscript. All authors critically reviewed the manuscript. All authors read and approved the final version of the manuscript.

\section{Funding}

The study was funded by an intramural research grant

\section{Compliance with ethical standards}

\section{Conflict of interest}

The authors declare no competing interests.

\section{Ethics approval}

The study was approved by the Ethics Committee of our Institutional Review Board (IRB Min No: 9628, dated 01.09.2015). Informed consent was obtained from all adult patients. In the case of minors, assent was obtained from patients along with the informed consent of their parents / guardians.

\section{Availability of data and material}

On request

\section{References}

1. M. Reincke, S. Sbiera, A. Hayakawa, M. Theodoropoulou, A. Osswald, F. Beuschlein, T. Meitinger, E. Mizuno-Yamasaki, K. Kawaguchi, Y. Saeki, K. Tanaka, T. Wieland, E. Graf, W. Saeger, C. L. Ronchi, B. Allolio, M. Buchfelder, T. M. Strom, M. Fassnacht, and M. Komada, Mutations in the deubiquitinase gene USP8 cause Cushing's disease. Nat. Genet. 47, 31-38 (2014). https://doi.org/10.1038/ng.3166

2. L. G. Perez-Rivas, M. Theodoropoulou, F. Ferraù, C. Nusser, K. Kawaguchi, C. A. Stratakis, F. Rueda Faucz, L. E. Wildemberg, G. Assié, R. Beschorner, C. Dimopoulou, M. Buchfelder, V. Popovic, C. M. Berr, M. Tóth, A. I. Ardisasmita, J. Honegger, J. Bertherat, M. R. Gadelha, F. Beuschlein, G. Stalla, M. Komada, M. Korbonits, and M. Reincke, The gene of the ubiquitin-specific protease 8 is frequently mutated in adenomas causing Cushing's disease. J. Clin. Endocrinol. Metab. 100, E997-1004 (2015). https://doi.org/10.1210/jc.2015-1453

3. Z.-Y. Ma, Z.-J. Song, J.-H. Chen, Y.-F. Wang, S.-Q. Li, L.-F. Zhou, Y. Mao, Y.-M. Li, R.-G. Hu, Z.-Y. Zhang, H.-Y. Ye, M. Shen, X.-F. Shou, Z.-Q. Li, H. Peng, Q.-Z. Wang, D.-Z. Zhou, X.-L. Qin, J. Ji, J. Zheng, H. Chen, Y. Wang, D.-Y. Geng, W.-J. Tang, C.-W. Fu, Z.-F. Shi, Y.-C. Zhang, Z. Ye, W.-Q. He, Q.-L. Zhang, Q.-S. 
Tang, R. Xie, J.-W. Shen, Z.-J. Wen, J. Zhou, T. Wang, S. Huang, H.-J. Qiu, N.-D. Qiao, Y. Zhang, L. Pan, W.-M. Bao, Y.-C. Liu, C.-X. Huang, Y.-Y. Shi, and Y. Zhao, Recurrent gain-of-function USP8 mutations in Cushing's disease. Cell Res. 25, 306-317 (2015). https://doi.org/10.1038/cr.2015.20

4. C. Ballmann, A. Thiel, H. E. Korah, A.-C. Reis, W. Saeger, S. Stepanow, K. Köhrer, G. Reifenberger, C. B. Knobbe-Thomsen, U. J. Knappe, and U. I. Scholl, USP8 Mutations in Pituitary Cushing AdenomasTargeted Analysis by Next-Generation Sequencing. J. Endocr. Soc. 2, 266-278 (2018). https://doi.org/10.1210/js.2017-00364

5. M. Losa, P. Mortini, A. Pagnano, M. Detomas, M. F. Cassarino, and F. Pecori Giraldi, Clinical characteristics and surgical outcome in USP8-mutated human adrenocorticotropic hormonesecreting pituitary adenomas. Endocrine 63, 240-246 (2018). https://doi.org/10.1007/s12020-0181776-0

6. K. Hayashi, N. Inoshita, K. Kawaguchi, A. Ibrahim Ardisasmita, H. Suzuki, N. Fukuhara, M. Okada, H. Nishioka, Y. Takeuchi, M. Komada, A. Takeshita, and S. Yamada, The USP8 mutational status may predict drug susceptibility in corticotroph adenomas of Cushing's disease. Eur. J. Endocrinol. 174, 213-226 (2016). https://doi.org/10.1530/EJE-15-0689

7. I. Weigand, L. Knobloch, J. Flitsch, W. Saeger, C. M. Monoranu, K. Höfner, S. Herterich, R. Rotermund, C. L. Ronchi, M. Buchfelder, M. Glatzel, C. Hagel, M. Fassnacht, T. Deutschbein, and S. Sbiera, Impact of USP8 Gene Mutations on Protein Deregulation in Cushing Disease. J. Clin. Endocrinol. Metab. 104, 2535-2546 (2019). https://doi.org/10.1210/jc.2018-02564

8. M. Riebold, C. Kozany, L. Freiburger, M. Sattler, M. Buchfelder, F. Hausch, G. K. Stalla, and M. PaezPereda, A C-terminal HSP90 inhibitor restores glucocorticoid sensitivity and relieves a mouse allograft model of Cushing disease. Nat. Med. 21, 276-280 (2015). https://doi.org/10.1038/nm.3776

9. J. Chen, X. Jian, S. Deng, Z. Ma, X. Shou, Y. Shen, Q. Zhang, Z. Song, Z. Li, H. Peng, C. Peng, M. Chen, C. Luo, D. Zhao, Z. Ye, M. Shen, Y. Zhang, J. Zhou, A. Fahira, Y. Wang, S. Li, Z. Zhang, H. Ye, Y. Li, J. Shen, H. Chen, F. Tang, Z. Yao, Z. Shi, C. Chen, L. Xie, Y. Wang, C. Fu, Y. Mao, L. Zhou, D. Gao, H. Yan, Y. Zhao, C. Huang, and Y. Shi, Identification of recurrent USP48 and BRAF mutations in Cushing's disease. Nat. Commun. 9, 3171 (2018). https://doi.org/10.1038/s41467-018-05275-5

10. S. Sbiera, L. G. Perez-Rivas, L. Taranets, I. Weigand, J. Flitsch, E. Graf, C.-M. Monoranu, W. Saeger, C. Hagel, J. Honegger, G. Assie, A. R. Hermus, G. K. Stalla, S. Herterich, C. L. Ronchi, T. Deutschbein, M. Reincke, T. M. Strom, N. Popov, M. Theodoropoulou, and M. Fassnacht, Driver mutations in USP8 wild-type Cushing's disease. Neuro-Oncol. 21, 1273-1283

(2019). https://doi.org/10.1093/neuonc/noz109

11. S. Sarkar, S. Rajaratnam, G. Chacko, S. Mani, A. S. Hesargatta, and A. G. Chacko, Pure endoscopic transsphenoidal surgery for functional pituitary adenomas: outcomes with Cushing's disease. Acta Neurochir. (Wien) 158, 77-86 (2016). https://doi.org/10.1007/s00701-015-2638-7

12. M. Ferchichi, R. Jouini, I. Ayari, W. Koubaa, A. Chadli-Debbiche, and E. BenBrahim, KRAS, NRAS and BRAF analysis of ampullary adenocarcinoma classified using CK7, CK20, MUC1 and MUC2. J. Gastrointest. Oncol. 9, 820-827 (2018). https://doi.org/10.21037/jgo.2018.05.03 
13. F. R. Faucz, A. Tirosh, C. Tatsi, A. Berthon, L. C. Hernández-Ramírez, N. Settas, A. Angelousi, R. Correa, G. Z. Papadakis, P. Chittiboina, M. Quezado, N. Pankratz, J. Lane, A. Dimopoulos, J. L. Mills, M. Lodish, and C. A. Stratakis, Somatic USP8 Gene Mutations Are a Common Cause of Pediatric Cushing Disease. J. Clin. Endocrinol. Metab. 102, 2836-2843

(2017). https://doi.org/10.1210/jc.2017-00161

14. A. Albani, L. G. Pérez-Rivas, C. Dimopoulou, S. Zopp, P. Colón-Bolea, S. Roeber, J. Honegger, J. Flitsch, W. Rachinger, M. Buchfelder, G. K. Stalla, J. Herms, M. Reincke, and M. Theodoropoulou, The USP8 mutational status may predict long-term remission in patients with Cushing's disease. Clin. Endocrinol. (Oxf.) 89, 454-458 (2018). https://doi.org/10.1111/cen.13802

15. S. Sbiera, M. Kunz, I. Weigand, T. Deutschbein, T. Dandekar, and M. Fassnacht, The New Genetic Landscape of Cushing's Disease: Deubiquitinases in the Spotlight. Cancers 11, 1761 (2019). https://doi.org/10.3390/cancers11111761

16. L. G. Pérez-Rivas, M. Theodoropoulou, T. H. Puar, J. Fazel, M. R. Stieg, F. Ferraù, G. Assié, M. R. Gadelha, T. Deutschbein, M. C. Fragoso, B. Kusters, W. Saeger, J. Honegger, M. Buchfelder, M. Korbonits, J. Bertherat, G. K. Stalla, A. R. Hermus, F. Beuschlein, and M. Reincke, Somatic USP8 mutations are frequent events in corticotroph tumor progression causing Nelson's tumor. Eur. J. Endocrinol. 178, 57-63 (2018). https://doi.org/10.1530/EJE-17-0634

17. L. G. Libuit, A. S. Karageorgiadis, N. Sinaii, N. M. Nguyen May, M. F. Keil, M. B. Lodish, and C. A. Stratakis, A gender-dependent analysis of Cushing's disease in childhood: pre- and postoperative follow-up. Clin. Endocrinol. (Oxf.) 83, 72-77 (2015). https://doi.org/10.1111/cen.12644

18. S. S. Chaidarun, B. Swearingen, and J. M. Alexander, Differential expression of estrogen receptor-beta (ER beta) in human pituitary tumors: functional interactions with ER alpha and a tumor-specific splice variant. J. Clin. Endocrinol. Metab. 83, 3308-3315

(1998). https://doi.org/10.1210/jcem.83.9.5128

19. S. Oomizu, J. Honda, S. Takeuchi, T. Kakeya, T. Masui, and S. Takahashi, Transforming growth factor-alpha stimulates proliferation of mammotrophs and corticotrophs in the mouse pituitary. J. Endocrinol. 165, 493-501 (2000). https://doi.org/10.1677/joe.0.1650493

20. E. H. Oldfield and A. O. Vortmeyer, J. Development of a histological pseudocapsule and its use as a surgical capsule in the excision of pituitary tumors. Neurosurg. 104, 7-19 (2006). https://doi.org/10.3171/jns.2006.104.1.7

\section{Tables}

Due to technical limitations, table 1 to 6 are only available as a download in the Supplemental Files section.

\section{Figures}


TACTC CTC CC CAGATATAAC

||$_{A A 718}||_{\text {AA } 719}$

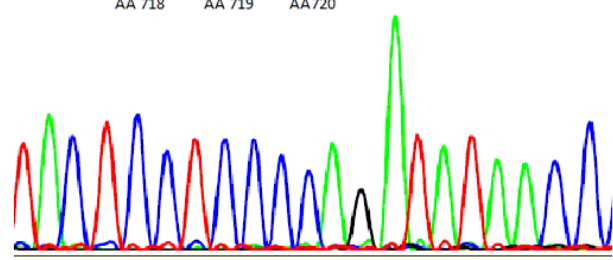

a

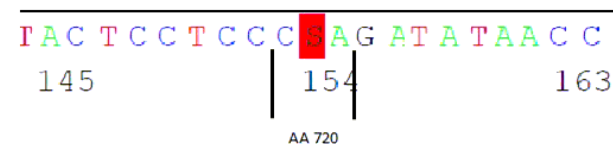

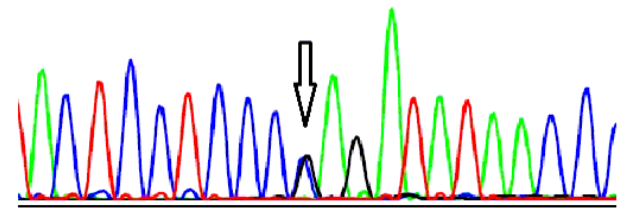

b
GC T C T AC T C C CMCMNATA 145

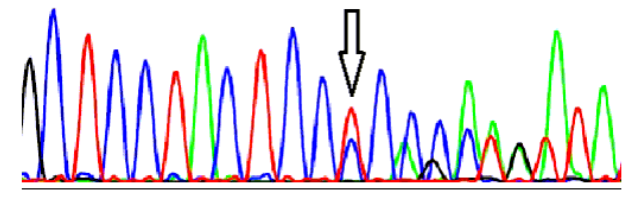

C

Figure 1

Fig. 1a - Sequencing chromatogram of a USP8 wild-type tumour Fig. 1b - Sequencing chromatogram showing a p.Pro720Arg mutation of USP8 Fig. 1c - Sequencing chromatogram showing a p.Ser719del fs mutation of USP8

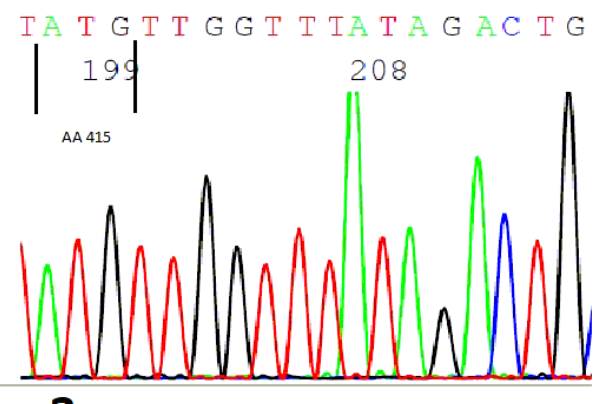

a
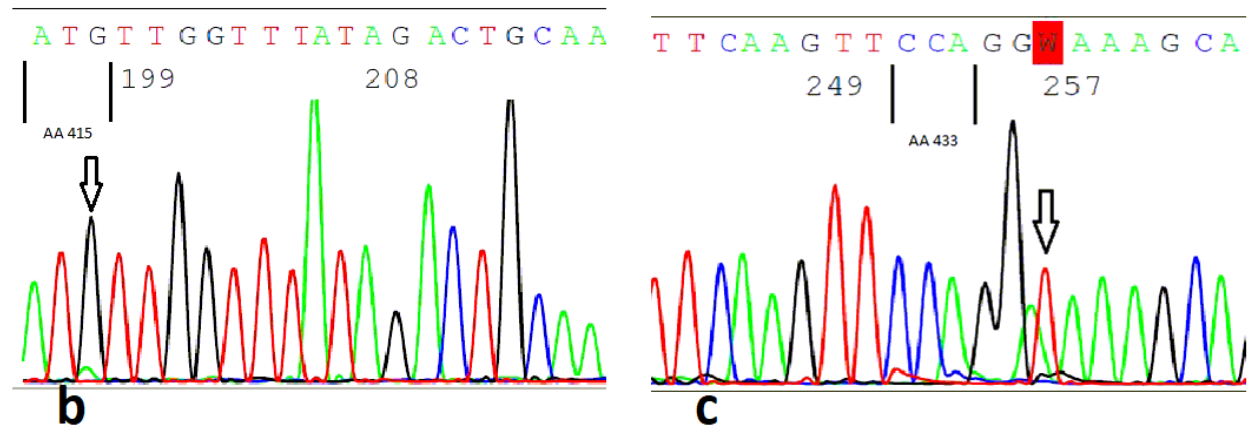

Figure 2

Fig. 2a - Sequencing chromatogram of USP48 wild-type tumour Fig. 2b - Sequencing chromatogram showing a p.Met415lle mutation of USP48 Fig. 2c - Sequencing chromatogram showing a USP48 splicesite mutation (p.Pro433IVS+2 T>A)

\section{Supplementary Files}

This is a list of supplementary files associated with this preprint. Click to download.

- Tables.docx

- Supplementarymaterial.docx 